(C) [2009] IEEE. Reprinted, with permission, from Xu, Wei; Zhu, Jianguo; Guo, Youguang; Tan, Longcheng; Wang, Shuhong. 2009, 'Analysis on Performance of a Linear Induction Motor based on Winding Function Method', Proceedings of the 4th IEEE Conference on Industrial Electronics and Applications, pp. 3639-3642. This material is posted here with permission of the IEEE. Such permission of the IEEE does not in any way imply IEEE endorsement of any of the University of Technology, Sydney's products or services. Internal or personal use of this material is permitted. However, permission to reprint/republish this material for advertising or promotional purposes or for creating new collective works for resale or redistribution must be obtained from the IEEE by writing to pubs-permissions@ieee.org. By choosing to view this document, you agree to all provisions of the copyright laws protecting it. 


\title{
Analysis on Performance of Linear Induction Motor Basing on Winding Function Method
}

\author{
Wei $\mathrm{Xu}^{1}$, Jianguo Zhu ${ }^{1}$, Longcheng $\mathrm{Tan}^{2}$, Youguang Guo ${ }^{1}$, Shuhong Wang ${ }^{1,3}$ \\ (1.Faculty of Engineering, University of Technology, Sydney, NSW 2007, Australia \\ 2. Institute of Electrical Engineering Chinese Academy of Sciences, Beijing 100190, China \\ 3. Faculty of Electrical Engineering, Xi'an Jiaotong University, Xi'an 710049, China)
}

\begin{abstract}
Abastract- Winding function method is adopted in this paper basing on air-gap magnetic flux density equations, and a new equivalent circuit is presented so as to analyze single linear induction motor (SLIM) applied in linear metro. Steady and dynamic performances of SLIM can be predicted by this circuit considering end effects, half filled slots, saturated iron and skin effect. To begin with, Dynamic situation under constant current constant frequency and several stable cases like constant current constant frequency or variable frequencies, constant voltage constant frequency or variable frequencies are presented in detail. Then, dynamic characteristics of SLIM under slip frequency control in both constant current and voltage drive regions are investigated. Furthermore, comparisons between conclusions on phase current, thrust, efficiency of high power SLIM and experimental results are made. The results indicate that winding function method is definitely reasonable to describe performance of SLIM, especially its end effects. It can be used in the design and performance prediction of SLIM.
\end{abstract}

\section{INTRODUCTION}

The single linear induction motor (SLIM) is proven as a better drive for urban transit vehicles, which is shown in Fig.1. Comparing to rotary induction motor (RIM) drive system, it could achieve more propulsive thrust independent on friction between wheel and rail. Furthermore, it has smaller turn circle radius, smaller sectional area of a tunnel, stronger climbing ability, lower noise, bigger acceleration and so on[1][2].

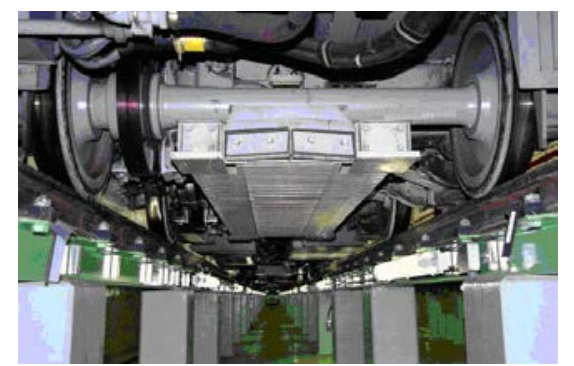

Fig.1 Simple vehicle system diagram drived by SLIM

The performance of SLIM is different from that of RIM. An accurate equivalent circuit model can be made due to its pole symmetry in RIM. Otherwise, there is no similar recognized model for SLIM because some parameters like mutual inductance could change by velocity. For its special structure, the SLIM has transverse and longitudinal end effects. The former resulted from different width between primary and secondary can bring some deformation in transverse air flux density so as to increase the secondary resistivity. The latter resulted from the discontinuous magnetic circuit and electrical condition change at the end points in moving direction can reduce its mutual inductance and net thrust. Moreover, when a double primary layer winding is adapted, a problem named half-filled slots occurs near both ends for the cut-open circuit.

Some papers are available on the analysis of SLIM performance. Ref. [1] has proposed a T-model equivalent circuit and corresponding correction coefficients for end effects, which could be applied in wide arrange. However, it is not suitable to control and dynamic analysis. Ref.[2] has gained one function according to secondary eddy current average and conversion energy balance, which is affected by speed and structure parameters. This method can be applied easily in SLIM control schemes widely. However, the process is very coarse, whose error would increase as the velocity goes up. Ref. [3] can theoretically simulate stable and dynamic performance by space harmonic method, but it requires more substantial computing time to gain the useful results.

This paper sets out a new model, which can be used conveniently to analyze steady and dynamic performance of SLIM. Winding function method is also used to calculate circuit parameters, such as mutual inductance, secondary resistance. More detail is introduced below.

\section{EQUIVALENT CIRCUIT MODEL OF SLIM}

From Ampere's law, Faraday's law and Maxwell law, the air-gap flux density equation of SLIM can be drawn below,

$$
\frac{\partial^{2} b_{y}}{\partial x^{2}}-\sigma \mu_{0} V \frac{\partial b_{y}}{\partial x}-j \sigma \mu_{0} \omega b_{y}=-j \mu_{0} \frac{\pi}{\tau} J_{1} e^{j(\omega t-\pi x / \tau)}(1)
$$

Where, $\sigma$ is secondary conductivity, $\mu_{0}$ is air permeability. The solution of $\mathrm{b}_{\mathrm{y}}(x, t)$ is,

$$
b_{y}(x, t)=\dot{B}_{0} e^{-j \frac{\pi x}{\tau}}+\dot{B}_{1} e^{-\frac{x}{\alpha_{1}}} e^{-j \frac{\pi x}{\tau_{e}}}+\dot{B}_{2} e^{\frac{x}{\alpha_{2}}} e^{j \frac{\pi x}{\tau_{e}}}
$$

Where, $b_{y}$ consists of three parts, $B_{0}, B_{1}$ and $B_{2}$. $B_{0}$ is normal traveling wave which travels forward like the 
fundamental flux density in RIM. $\mathrm{B}_{1}$ and $\mathrm{B}_{2}$, determined from boundary conditions, are entrance and exit end-effect waves. $\alpha_{1}, \alpha_{2}, \tau_{e}$ are functions of speed and motor structure parameters.

On the base of air-gap flux density equation, the paper divides secondary winding function of SLIM into fundamental component and end effect component. To begin with,it deduces two-phase stationary axis primary

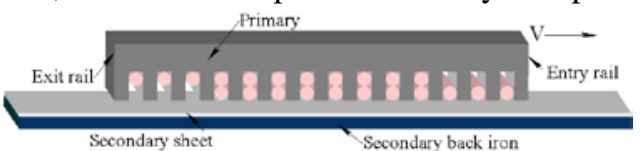

(a) Structure

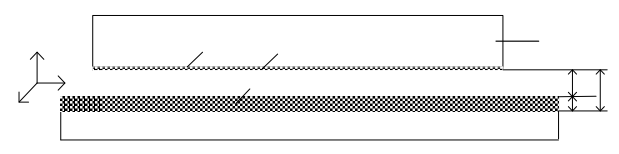

(b) Model

Fig. 2 The structure and analytic model of SLIM

on winding functions according to primary winding distribution. The secondary fundamental one and end effect one are gained separately from stable state and dynamic state of gap magnetic flux equations. Moreover, it calculates all inductances, goodness factor, secondary resistor and speed voltage coefficients, then achieves voltage and flux equations. On the energy conversion relationship between primary and secondary, it gets the expressions of thrust, power factor and efficiency. In the whole, the whole derivation progress is a little complex, more details are shown in [4][6]. A summary of this model is presented below.

The primary three-static-axis winding functions,

$$
\left\{\begin{array}{l}
N_{a s}(x)=\frac{N_{s}}{2} \cos (\pi x / \tau+\pi) \\
N_{b s}(x)=\frac{N_{s}}{2} \cos (\pi x / \tau+\pi / 3) \\
N_{c s}(x)=\frac{N_{s}}{2} \cos (\pi x / \tau-\pi / 3)
\end{array}\right.
$$

Where, $N_{\mathrm{s}}$ is the fundamental part per pole winding series.

The primary two-static-axis winding functions,

$$
\left\{\begin{array}{l}
N_{\alpha s}(x)=\frac{3}{4} N_{s} \sin (\pi x / \tau) \\
N_{\beta s}(x)=-\frac{3}{4} N_{s} \cos (\pi x / \tau)
\end{array}\right.
$$

The secondary fundamental part winding functions,

$$
\left\{\begin{array}{l}
N_{\alpha r s}(x)=\frac{3}{4} N_{s} \sin (\pi x / \tau) \\
N_{\beta r s}(x)=-\frac{3}{4} N_{s} \cos (\pi x / \tau)
\end{array}\right.
$$

The secondary end effect part winding functions,

$$
\left\{\begin{array}{l}
N_{\text {are }}(x)=-\frac{N_{2}}{K} \exp \left(-x / \alpha_{2}\right) \sin \left(\pi x / \tau_{e}-\theta_{e}\right) \\
N_{\beta r e}(x)=\frac{N_{2}}{K} \exp \left(-x / \alpha_{2}\right) \cos \left(\pi x / \tau_{e}-\theta_{e}\right)
\end{array}\right.
$$

where $N_{2}, \theta_{e}$ are related with slip frequency, goodness factor of SLIM.

The SLIM equation in matrix form is given by,

$$
\vec{u}=[R] \vec{i}+\frac{d \vec{\lambda}}{d t}+v \frac{\pi}{\tau}[U] \vec{\lambda}
$$

Where, vectors are expressed by,

$$
\vec{\lambda}=[L] \vec{i}
$$

$$
\begin{aligned}
& \vec{u}=\left[u_{\alpha s}, u_{\beta s}, 0,0\right]^{T} \\
& \vec{i}=\left[i_{\alpha s}, i_{\beta s}, i_{\alpha r}^{\prime}, i_{\beta r}^{\prime}\right]^{T} \\
& {[R]=\left[\begin{array}{cccc}
R_{s} & 0 & 0 & 0 \\
0 & R_{s} & 0 & 0 \\
0 & 0 & R_{r}^{\prime} & 0 \\
0 & 0 & 0 & R_{r}^{\prime}
\end{array}\right]} \\
& {[U]=\left[\begin{array}{cccc}
0 & 0 & 0 & 0 \\
0 & 0 & 0 & 0 \\
0 & 0 & 0 & 1 \\
0 & 0 & -1 & 0
\end{array}\right]}
\end{aligned}
$$

$L$ is inductance matrix, whose value between any two winding may be calculated by ,

$$
L_{12}=\frac{2 \mu_{0} l_{\delta}}{3 g_{e}} \int_{0}^{p \tau} N_{1}(x) N_{2}(x) d x
$$

Where, $N_{1}(\mathrm{x}), N_{2}(\mathrm{x})$ are winding functions, $l_{\delta}$ is primary stack width, $g_{\mathrm{e}}$ is equivalent air-gap width, $p$ is number of poles.

On the energy conversion balance between primary and secondary, the thrust is

$$
F_{e}=(3 \pi / 2 \tau)\left[i_{1}\right]^{T}[G]\left[i_{1}\right]
$$

Where, vector $i_{1}$ includes end effect part,

$$
\left[i_{1}\right]=\left[i_{\alpha s}, i_{\beta s}, i_{\alpha r}^{\prime}, i_{\beta r}^{\prime},\left(i_{\alpha s}+i_{\alpha r}^{\prime}\right),\left(i_{\beta s}+i_{\beta r}^{\prime}\right)\right]^{T}
$$

$G$ is speed voltage coefficient matrix, which is calculated like that of inductance $L$. 
The input active power,

$$
p_{\text {in }}=\frac{3}{2}\left(u_{\alpha s} i_{\alpha s}+u_{\beta s} i_{\beta s}\right)
$$

The input reactive power,

$$
q_{\text {in }}=\frac{3}{2}\left(u_{\beta s} i_{\alpha s}-u_{\alpha s} i_{\beta s}\right)
$$

The output active power,

$$
p_{\text {out }}=F_{e} v
$$

The power factor,

$$
\cos \varphi=p_{\text {in }} / \sqrt{{p_{\text {in }}{ }^{2}+{q_{i n}}^{2}}^{2}}
$$

The efficiency,

$$
\eta=p_{\text {out }} / p_{\text {in }}
$$

\section{DYNAMIC AND STEADY ANALYSIS}

The above derivations describe dynamic state of SLIM in differential form of linkage. The equations could be solved by numerical step by step method. In most dynamic cases, the state variables include secondary linkages and primary currents.

The equations can also be readily used to steady-state analysis, which can be calculated by simply setting $d / d t$ to jw and solving equ. (7).

This paper have studied dynamic and steady states thrust curves of high power SLIM under current control or voltage control. The main parameters are shown in table I.

Fig. 3 is thrust curves calculated by steady and dynamic states equations. In the whole process, the primary current is controlled constant $280 \mathrm{~A}$, and primary frequency is constant $10 \mathrm{~Hz}$. It is apparent that the steady-state thrust are very close to the average of dynamic one. It is very interesting to observe double frequency $(20 \mathrm{~Hz})$ thrust pulsation as motor approaches. This is due mainly to the electric dynamics in the secondary circuits and produced by the end effects of SLIM.

Fig.4 is thrust curves calculated by stable state equations whose primary current is kept constant 280A. Dash lines indicate thrusts not considering end effects, but continuous lines do. Due to longer air gap and end effects, the thrust should decrease as velocity gets up [5].

Fig. 5 is thrust curves calculated by stable state equations whose primary phase voltage is kept constant $635 \mathrm{v}$. The trend of thrust likes that of fig. 4 .

The thrust of SLIM in transportation is most important. In actual SLIM control scheme, it often includes two regions such as "constant current” and “constant power" . Below the base speed, primary phase current and slip frequency are kept constant. Due to power conditioner voltage limit, constant voltage operation is encountered above base speed. The phase current decreases very quickly for the increasing total impedance. In order to meet with the operating requirement, it is necessary to increase slip frequency linearly so as to prevent quick reduction in thrust.

This paper by stable equations has analyzed the SLIM performance in the whole working area and compared simulation with measurement including phase current, thrust and efficiency. The base speed is $40 \mathrm{Km} / \mathrm{h}$. The phase current, thrust and efficiency curves are shown separately in Fig.6, Fig.7 and Fig.8.

$$
\text { TABLE I }
$$

\begin{tabular}{|c|c|c|}
\hline \multicolumn{3}{|c|}{ THE DIMENSIONS OF THE SLIM } \\
\hline Pole pitch & Number of pole pairs & Primary lengh/width \\
\hline $0.2808(\mathrm{~m})$ & 4 & $2.476 / 0.3(\mathrm{~m})$ \\
\hline Secondary width & Secondary sheet thin & Air gap \\
\hline $0.36(\mathrm{~m})$ & $7 \mathrm{~mm}$, Copper & $9(\mathrm{~mm})$ \\
\hline Base frequency & Thrust & Primary phase voltage \\
\hline $22.5(\mathrm{~Hz})$ & $25(\mathrm{KN})$ & $635(\mathrm{v})$ \\
\hline Line current & Number of slots & Secondary sheet width \\
\hline $280 \mathrm{~A}$ & 79 & $0.36 \mathrm{~m}$ \\
\hline
\end{tabular}

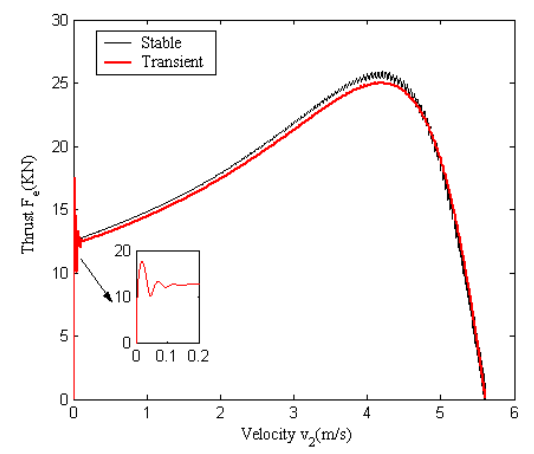

Fig.3 Thrust curves in constant current constant frequency calculated by stable and dynamic states

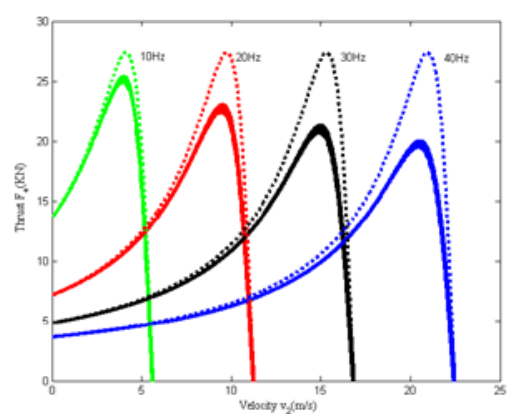

Fig.4 Thrust curves in constant current variable frequencies calculated by stable state 


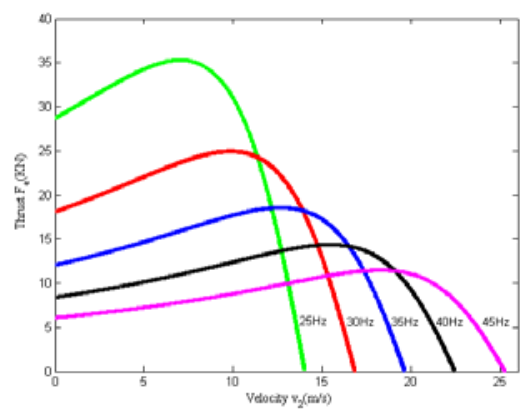

Fig.5 Thrust curves in constant voltage variable frequencies calculated

by stable state

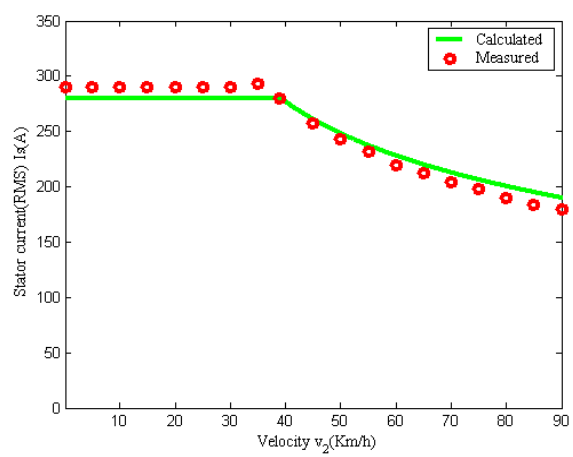

Fig.6 Primary phase current variable curves

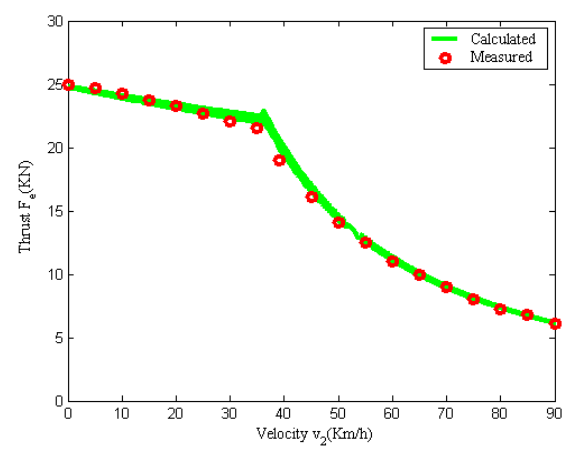

Fig.7 Thrust variable curves

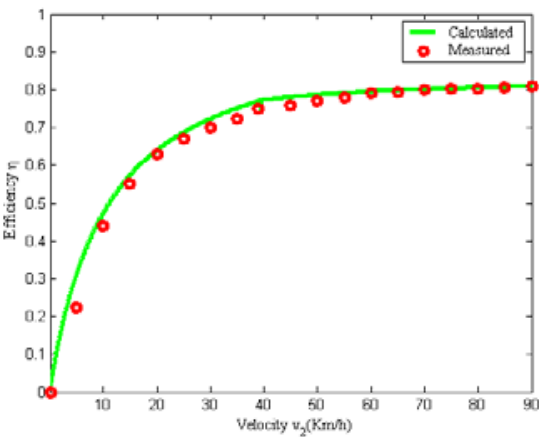

Fig.8 Efficiency variable curves

Simulation phase current in Fig.6 is close to measurement, which are kept constant below base speed and decreased linearly beyond that. Fig.7 indicates curves of thrust. Below base speed, the thrust decreases a little as speed goes up for end effect although the phase current is constant. Beyond base speed, thrust decreases linearly for the reducing phase current. Error in base speed is obvious because control manner and slip frequency have changed. Fig.8 is curves of efficiency. The calculation and measurement agree with each other, which meet requirement of engineering application.

\section{CONCLUSIONS}

This paper has set out a new equivalent circuit of SLIM basing on winding function method. The end effects are automatically included in machine parameters. It can analyze steady and dynamic states of SLIM in a similar way as for RIM. Simulation results agree with experiment very well, which make the circuit a very useful tool for a general research of dynamic and control problems in SLIM.

\section{ACKNOWLEDGEMENT}

The authors are grateful to Prof. Yaohua $\mathrm{Li}$ in the Institute of Electrical Engineering, Chinese Academy of Sciences for his helpful suggestions.

\section{REFERENCES}

[1] Xialing Long, Theory and magnetic design method of linearinduction motor,China: Science publishing company,2006,pp.50-100

[2] G. Kang, K. Nam, “Field-oriented control scheme for linear induction motor with the end effect," IEE Proceeding on Electrical Power Application. Vol.152,No.6, pp.1565-1572, November 2005.

[3] Tsuyoshi Higuchi, Sakutaro Nonaka. "On the design of high efficiency linear induction motors for linear metro," Electrical Engineering in Japan,Vol.137, No.2,pp36-43,August 2001.

[4] Changan Lu, "A new coupled-circuit model of a linear induction motor and its application to steady-state, dynamic ,dynamic and control studies," thesis of Queen university, Canada, 1993, pp.22-27

[5] Xu Wei, Sun Guangsheng, Li Yaohua, “A new parameter measurement scheme for single linear induction motor,” Transactions of China Electro Technical Society,Vol.22, No.6,pp54-58,June 2007.

[6] Wei Xu, "Research on the Performance of Single-sided Linear Induction Motor," thesis of Chinese academy of sciences, China, 2008, pp.40-80 